\title{
Sakrale Nervenstimulation im Kindesalter
}

\section{Die Standardbehandlung von Kindern mit einer Blasen- funktionsstörung umfasst verhaltenstherapeutische sowie} pharmakologische Ansätze. Die zur Therapie der überaktiven Blase (OAB) im Erwachsenenalter etablierte sakrale Nervenstimulation (SNS) stellt auch im Kindesalter eine vielversprechende Behandlungsoption dar. Eine US-amerikanische Arbeitsgruppe der Ohio State University hat ein standardisiertes SNS-Behandlungs- und Nachsorgeprotokoll für Kinder mit einer therapierefraktären Blasendysfunktion entwickelt und das subjektive und objektive Therapieansprechen der Patienten evaluiert.

J Urol 2015; 194: 1721-1727

\section{mit Kommentar}

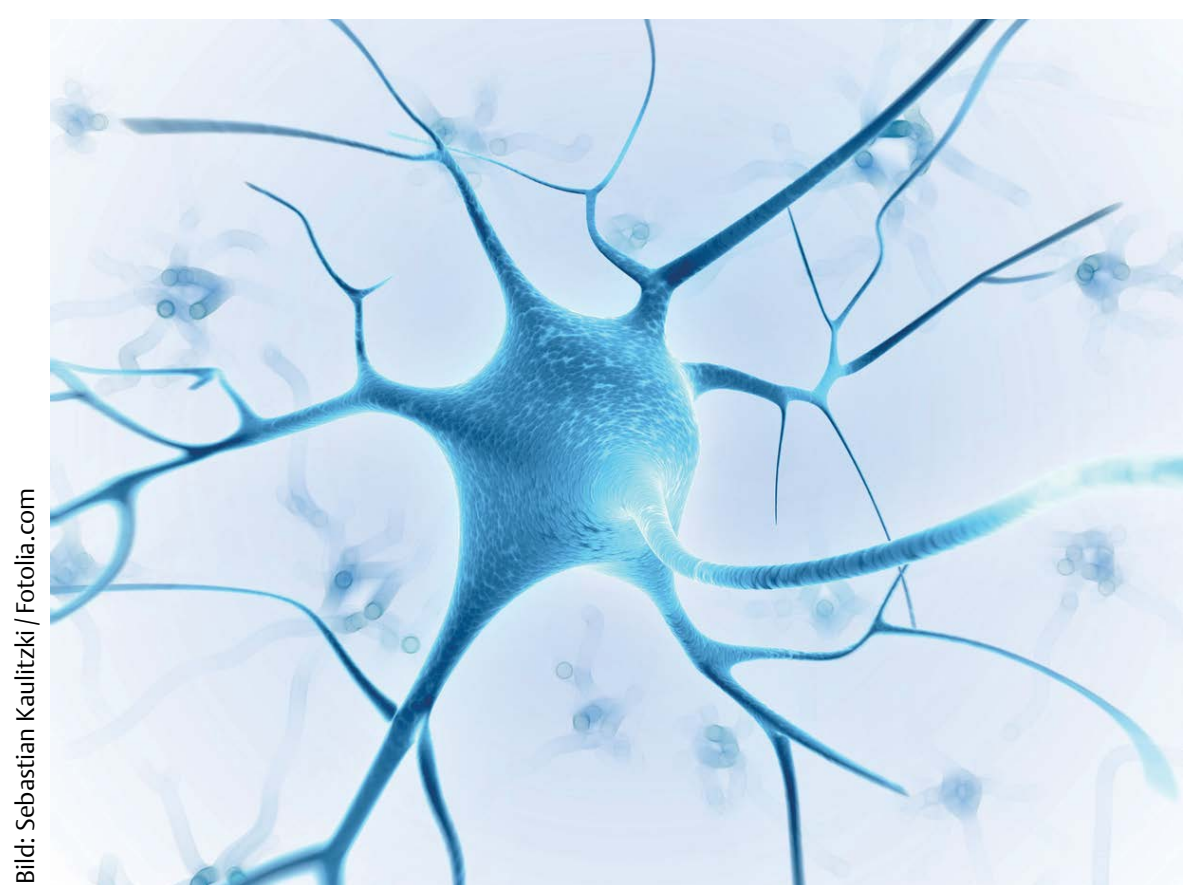

An der Studie nahmen zwischen 2012 und 201526 Kinder (durchschnittliches Alter 10,8 Jahre, Range 6,1-17,3) mit einer schweren, therapierefraktären primären Blasenfunktionsstörung teil. In 22 Fällen lag eine OAB-Symptomatik mit oder ohne Inkontinenzproblematik vor. Drei Patienten litten an einer Detrusorunterfunktion mit unvollständiger Blasenentleerung und Notwendigkeit zur intermittierenden Katheterisierung. In einem Fall lag eine kombinierte Blasenfunktionsstörung vor.
Vor der zweizeitigen chirurgischen Implantation eines SNS-Pulsgenerators im Bereich der Nervenwurzel S3 wurden alle Probanden mittels Urodynamik, Nierensonografie und spinaler Magnetresonanztomografie untersucht. Zudem beantworteten die Patienten den NLUTD / DES (Vancouver Non-neurogenic Lower Urinary Tract Dysfunction/Dysfunctional Elimination Syndrome Questionnaire), einen validierten Fragebogen zur Erfassung der Blasen- und Darmsymptomatik. Auch postoperativ wurden die Probanden regelmäßig urodynamisch sowie mittels Fragebogen-Evaluation nachbetreut (medianes Follow-up 1,2 Jahre; Range 0,34$2,20)$.

\section{Kontinenz bei $\mathbf{2 0} \%$ erreicht $\checkmark$}

Nach der Implantation des SNS-Impulsgebers war bei 66,7\% der Patienten mit OABSymptomatik $(n=23)$ eine signifikante Verbesserung des NLUTD/DES-Scores $(\mathrm{p}=0,0002)$ nachweisbar. Bei keinem Kind verschlechterte sich eine vorbestehende Inkontinenz, in $65 \%$ der Fälle trat eine Symptombesserung ein, und in $20 \%$ der Fälle wurde postoperativ eine Kontinenz erreicht. Auch der durchschnittliche Enuresis-Score verbesserte sich in der OAB-Gruppe postoperativ signifikant $(p=0,018)$. Mithilfe der urodynamischen Verlaufsbeobachtung ließ sich in der OABGruppe postoperativ eine signifikante $A b$ nahme der Anzahl ungehemmter Detrusorkontraktionen sowie eine signifikante Abnahme des maximalen Detrusordrucks in der Füllungsphase nachweisen ( $p=0,016$ bzw. $p=0,024)$. Diese urodynamischen Parameter korrelierten mit der subjektiven Symptombesserung.

In der Gruppe der Kinder mit gestörter Blasenentleerung $(n=4)$ ließ sich nach der Implantation des SNS-Impulsgebers im Vergleich zu den präoperativen Messwerten eine deutliche Abnahme der durchschnittlichen Restharnmenge nach Miktion beobachten ( 235,5 vs. $765,25 \mathrm{ml}$; $\mathrm{p}=0,04)$. In 3 Fällen verbesserte sich auch die maximale Harnflussrate, diese Zunahme war jedoch statistisch nicht signifikant. Bei allen Patienten sank die Katheterisierungsfrequenz.

\section{Fazit \\ Sowohl Kinder mit einer überaktiven als auch Kinder mit einer unteraktiven, the- rapierefraktären Blasenfunktionsstö- rung, so das Fazit der Autoren, profitie- ren hinsichtlich der subjektiven Sympto- matik sowie der objektiv messbaren urodynamischen Parameter von der Im- plantation eines SNS-Impulsgebers. Das langfristige Outcome der mittels sakra- ler Nervenstimulation behandelten Pa- tienten, so ihre Empfehlung, müsse im Rahmen zukünftiger Studien analysiert werden.}

Dr. Judith Lorenz, Künzell 


\section{Erfolgversprechende Therapieoption?}

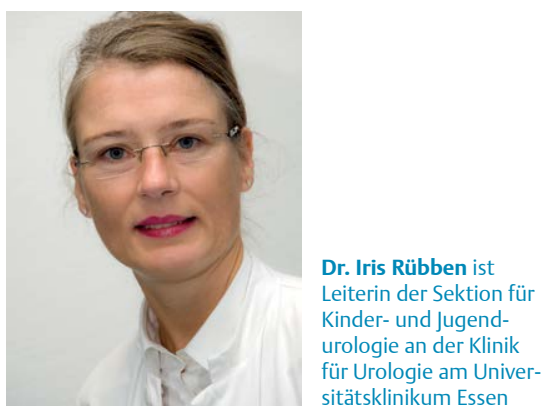

Kinderurologie, das bedeutet nicht nur gutes operatives Versorgungsmanagement, sondern in hohem Maße auch empathische Fachkompetenz in der Behandlung kindlicher Ausscheidungsstörungen, insbesondere der kindlichen Inkontinenz (nicht monosymptomatische Enuresis nocturna, NMEN).

Doch was bieten wir Eltern und Patienten an, wenn nach langwieriger Behandlung mit unterschiedlichen Therapiemodalitäten, wie urotherapeutische Instruktion, Physiotherapie und Biofeedback, regelmäßige Gabe von Medikamenten in Mono- oder Kombinationstherapie, keine zuverlässige Kontinenz erzielt werden konnte und die diagnostische Urethrozystoskopie, urodynamische Evaluation und das MRT der Neuroachse keine wegweisende Pathologie, bis auf die persistierenden Symptome einer überaktiven Blase, ermittelte?

Bis zu 20\% der Kinder mit einer OAB-Symptomatik betrifft diese Therapieresistenz [1-3].

Welche therapeutischen Optionen bleiben dieser speziellen Gruppe von Patienten und Eltern, deren Lebensqualität signifikant eingeschränkt ist [4], die im Alltagsleben in höherem Maße Mobbing ausgesetzt sind als ihre nicht betroffenen Altersgenossen [5], und die auch als Erwachsene häufiger Blasen- und Darmfunktionsstörungen aufweisen [6], wenn unsere konservativen Maßnahmen ausgeschöpft sind?

Die Autoren gehen in ihrer Publikation der Frage nach, ob bei diesen therapierefraktären Patienten (23 Patienten, mittleres Alter 10,8 Jahre, mittlerer Beobachtungszeitraum 1,2 Jahre) die sakrale Neuromo- dulation eine wirksame Therapieoption ist. Hierzu wurde in standardisiertem 2-stufigem Vorgehen den Patienten mit Beschwerden i.S.e. überaktiven Blase (Urgency-Frequency-Syndrom, Inkontinenz) ein sakraler Neuromodulator implantiert.

Diesen erhielten in der o.g. Untersuchung auch 3 Patienten mit einem hypotonen Detrusor (Detrusor-Unteraktivität) mit Restharnbildung, die katheterisierungspflichtig war. Aufgrund der sehr geringen Fallzahl wird in diesem Kommentar auf diese spezielle Patientengruppe nicht eingegangen.

In der vielzähligen Literatur zur funktionellen kindlichen Blasenfunktionsstörung fällt bei besonderer Betrachtung der überaktiven Blase auf, dass verschiedene erweiterte Therapieangebote bei primärer Therapieresistenz zu nahezu analogen Ansprechraten führen. So konnten intensivierte urotherapeutische Trainingsprogramme mit stationärer Betreuung [3] oder kombinierte konservative Behandlungsmodalitäten aus Standardurotherapie ergänzt mit transkutaner Neuromodulation (TENS) [7] in 75-80\% eine Symptomverbesserung bis Symptomfreiheit erzielen, während für 20-25\% der betroffenen Patienten weiterhin kein Ansprechen nachgewiesen werden konnte.

Die Injektion von 100 IE Botulinum-AToxin in die Detrusormuskulatur [8] kann für $70 \%$ bis dahin therapierefraktäre $\mathrm{Pa}$ tienten nach einer Injektion eine signifikante langfristige Verbesserung der Beschwerden erreichen - während auch nach dieser invasiven Behandlung etwa bei $15 \%$ der kindlichen OAB-Patienten die Symptome unbeeinflusst bleiben.

Nach Implantation eines sakralen Neuromodulators ergab sich in der o. g. Untersuchung für 35\% der Patienten keine Veränderung ihrer Inkontinenz-Symptomatik, während $20 \%$ mittels sakraler Neuromodulation kontinent wurden.

Betrachtet man Daten zum Placebo-Effekt in dieser Patientengruppe [9] wird die Einschätzung schwierig, ob mit 20\% Trockenheit in einem aufwendigen Therapiealgorithmus die sakrale Neuromodulation als eine erfolgversprechende Therapieoption in unseren kinderurologischen Alltag Eingang finden sollte.

Halten wir uns ferner vor Augen, dass für Patienten und ihre Familien nicht verbes- serte urodynamische Messwerte, sondern allein eine subjektiv als verbessert empfundene Kontinenz Parameter sind, die über den Erfolg einer Therapie entscheiden, dürfte der Stellenwert einer sakralen Neuromodulation nicht gleichwertig zur Gabe von 100 IE Botulinum-A-Toxin anzusiedeln sein.

Zusammenfassend bleibt festzuhalten, dass mit einer konservativen Basistherapie für etwa $80 \%$ der kindlichen Patienten im Verlauf Kontinenz erzielt werden kann. Muss die Therapie in ca. 20\% augmentiert werden (z.B. als stationäre Schulung, zusätzliche Anwendung von TENS oder Botox) werden wiederum $70-80 \%$ positiv profitieren, während für etwa $20 \%$ keine Verbesserung der $\mathrm{OAB}$ erzielt werden kann. Ob für diese „mehrfach therapieresistenten“ Kinder und Jugendlichen eine Indikation zur sakralen Neuromodulation besteht, ist meiner Meinung nach im Gespräch mit Eltern und Patient vorsichtig auszuloten.

\section{Dr. Iris Rübben, Essen}

\section{Literatur}

1 Curran JM, Kaefer M, Peters C et al. The overactive bladder in childhood: Long-term results with conservative management. J Urol 2000; 163: 574-577

2 Hellerstein S, Zguta AA. Outcome of overactive bladder in children. Clin Pediatr 2003; 42: 553-556

3 Meijer EF, Nieuwhof-Leppink AJ, DekkerVasse E et al. Central inhibition of refractory overactive bladder complaints, results of an inpatient training program. J Ped Urol 2015; 11: 21.e1-e5

4 Equit M, Hill J, Hübner A et al. Health-related quality of life and treatment effects on children with functional incontinence, and their parents. Pediatr Urol 2014; 10: 922-928

5 Zhao PT, Velez D, Faiena I et al. Bullying has a potential role in pediatric lower urinary tract symptoms. J Urol 2015; 193: 17431748

6 Bower WF, Yip SK, Yeung CK. Dysfunctional elimination symptoms in childhood and adulthood.; J Urol 2005; 174: 1623-1628

7 Sillén U, Arwidsson C, Doroszkiewicz M et al. Effects of transcutaneous neuromodulation (TENS) on overactive bladder symptoms in children: Arandomized controlled trial.J Ped Urol 2014; 10: 1100-1105

8 Hoebeke P, De Caestecker K, Vande Walle J et al. The effect of Botulinum-A Toxin in incontinent children with therapy resistant overactive detrusor. J Urol 2006; 176: 328-331

9 Boudaoud N, Binet A, Line A et al. Management of refractory overactive bladder in children by transcutaneous posterior tibial nerve stimulation: A controlled study. J Ped Urol 2015; 11: 138e1-138e10 